\title{
A Context-Aware Trust-Oriented Influencers Finding in Online Social Networks
}

\author{
Feng Zhu ${ }^{1,4}$, Guanfeng Liu ${ }^{1,4}$, Yan Wang ${ }^{2}$, An Liu ${ }^{1.4}$ Zhixu Li $^{1,4}$, Pengpeng Zhao ${ }^{1,4}$, Lei $\mathrm{Li}^{3}$, \\ ${ }^{1}$ School of Computer Science and Technology \\ Soochow University, China 215006 \\ ${ }^{2}$ School of Information Technology and Electrical Engineering \\ University of Queensland, QLD, Australia 4072 \\ ${ }^{3}$ School of Computer and Information, Heifei University of Technology, China 230009 \\ ${ }^{4}$ Collaborative Innovation Center of Novel Software Technology and Industrialization, Nanjing, China \\ Email: \{gfliu, anliu,zhixuli,ppzhao\}@suda.edu.cn; yan.wang@mq.edu.au; lilei@hfut.edu.cn
}

\begin{abstract}
Online Social Networks (OSNs) have been used as the means for a variety of applications, like employment system, e-Commerce and CRM system. In these applications, social influence acts as a significant role, affecting people's decisionmaking. However, the existing social influence evaluation methods do not fully consider the social contexts, like the social relationships and the social trust between participants, and the preferences of participants, which have significant impact on social influence evaluation in OSNs. Thus, these existing methods cannot deliver accurate social influence evaluation results. In our paper, we propose a Context-Aware TrustOriented Influencers Finding method, called CT-Influence, with social contexts taken into account. We conduct experiments onto two real social network datasets, i.e., Epinions and $D B L P$. The experimental results illustrate that our CT-Influence method greatly outperforms the state-of-the-art method SoCap in terms of effectiveness and efficiency.
\end{abstract}

Keywords-Social network; Social influence; Trust;

\section{INTRODUCTION}

\section{A. Background}

Online Social Networks (OSNs) are becoming more and more popular and have been used as the means in a variety of applications, like employment, CRM and e-Commerce. In these applications, the social influence of a participant can affect others' decision-making [1], [2]. For example, at Epinions (epinions.com), an OSN based e-commerce platform, a buyer can write a product review to rate the products and corresponding seller. This review can be viewed by other buyers and thus can impact their decision making in purchasing the same products. As indicated in studies of Social Psychology [3], [4], [5] and Computer Science [6], [7], [8], a person is more likely to accept the recommendations given by participants with higher social influence (named as Influencers) in a specific domain. Therefore, it is significant to accurate evaluate the social influence of participants and identify those Influencers from social networks

In the literature, many social influence evaluation methods have been proposed [9], [10], [11], [12], [13], [14], [15], [16], [17], [18], in which, Independent Cascade (IC) model [9] is a typical model to find the Top- $K$ nodes who have the maximal social influence in a network. Subsequently,

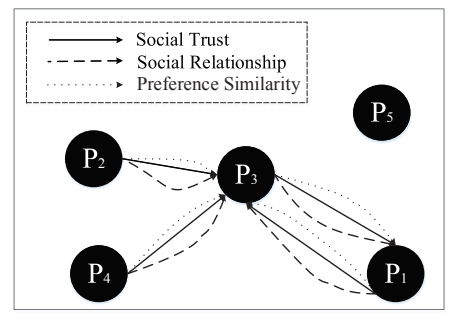

Figure 1. A social network from Epinions

some important works [15], [13] are proposed to improve the scalability of $I C$ model. In addition, in recent years, the $L o$ cal Influence Maximization method [19] has been proposed to evaluate the social influence of a specific participant in OSNs. Furthermore, as some OSNs are becoming a large real-time generator of social data-streams, some streaming methods [20], [21] have proposed to evaluate the social influence of participants in OSNs.

\section{B. The Problem and Motivation}

As illustrated in Social Psychology [22], [23], [24], the social trust between participants (e.g., students trust their lecturers in a specific research area), the social relationship between participants (e.g., the relationship between a father and his song), and the preference similarity between participants (e.g., they all like to play basketball) have significant influence on participants' decision-making, and thus impact their social influence. However, these important social contexts are not fully considered by the existing social influence evaluation methods. Thus, these methods cannot deliver accurate social influence evaluation results.

Example 1: Figure 1 depicts a social network from Epinions, which contains five participants (i.e., $P_{1}$ to $P_{5}$, they are all buyers). The trust relationship (represented as arrows with solid lines) between $P_{1}$ and $P_{3}, P_{2}$ and $P_{3}$, and $P_{4}$ and $P_{3}$ can be established based on the quality of the product review of $P_{3}$. Their social relationship and preferences can be mined from the their profiles and purchase history [25]. Suppose $P_{1}$ has closer social relationships, and has more similar preferences to $P_{3}$ than that of $P_{2}$, then $P_{3}$ can more likely affect the purchasing behavior of $P_{1}$ than $P_{2}$, which 
is not identified by the existing social influence evaluation methods.

The above mentioned problems motivate us to develop a social influence evaluation method to accurately evaluate participants' social influence in OSNs. In this paper, with considering the above mentioned important social contexts, we propose a Context-Aware Trust-Oriented Influencers Finding method, called CT-Influence by adopting iterative method. Since our method is convergent fast, thus we can deliver accurate social influence evaluation results with good efficiency.

\section{Contributions}

The main contributions of this paper can be summarised as follows:

- To the best of our knowledge, this is the first work that fully takes the social contexts into account in social influence evaluation.

- We propose a novel social influence evaluation method, CT-Influence, which achieves $\mathcal{O}\left(\lambda N^{2}\right)$ in computation cost, where $N$ is the number of nodes in an OSN and $\lambda$ is the iterative times in computation.

- We have conducted experiments on two real social network datasets, i.e., Epinions and DBLP. By comparing with the state-of-the-art individual social influence evaluation method, SoCap [16], our CT-Influence method greatly outperforms SoCap in effectiveness and efficiency for social influence evaluation.

\section{RELATED WORK}

In the literature, existing social influence evaluation approaches can be categorized into four groups as below.

\section{A. Global Influence Maximization}

The global influence maximization is to find a group of nodes that can impact the maximal number of other nodes in an OSN. Kempe et al. [9] propose a greedy algorithm which guarantees $(1-1 / e)$ approximation ratio. However, this algorithm has low efficiency in practice and thus it is not scalable with the network size. In order to improve the scalability, [13] propose an algorithm that has a simple turnable parameter, for users to control the balance between the running time and the influence spread of the algorithm. Jung et al. [11] propose an algorithm IRIE that integrates the advantages of influence ranking $(I R)$ and influence estimation (IE) methods for the global influence maximization. [14] provide a scalable influence approximation algorithm, Independent Path Algorithm (IPA), for $I C$ model. In the model, they study $I P A$ efficiently approximates influence by considering an independent influence path as an influence evaluation unit. Moreover, in order to spend up the evaluation algorithm, [15] develop the $C E L F$ algorithm, which exploits sub-modularity to find near-optimal influencer selections.

\section{B. Local Influence Maximization}

The local influence maximization is to find a group of nodes that have the maximal impacts on a specified participant. Yeung et al. [17] have studied the relations between trust and product ratings in online consumer review sites. Moreover, they propose a method to estimate the strengths of trust relations so as to estimate the true influence among the trusted participants. In addition, Guo et al. [19] propose a method to find $K$ nodes that have the maximal impacts on a specified participant. Furthermore, Iwata et al. [26] propose a probabilistic model to discover the latent influence between participants in OSNs. The model is used to find influential participants and discover relations between participants.

\section{Stream Learning of Influence}

In recent years, $O S N$ is becoming a large real-time generator of social data-streams, like Twitter (twitter.com). The streaming methods of social influence become more and more popular. Kutzkov et al. [20] propose a streaming method, called STRIP for computing the influence strength along each link of an $O S N$. In addition, Karthik et al. [21] propose an approach to mine the flow patterns, following specific flow validity constraints. However, contrasting with microblogging platforms, the other OSNs cannot provide sufficient contexts to perform information flow pattern discovery. Thus, the streaming methods cannot be applied for the social influencer finding in the OSN based e-commerce platforms.

\section{Individual Influence Evaluation Problem}

In order to evaluate the social influence of a specific participant, Subbian et al. [16] propose an approach, called SoCap, to find influencers in an OSN by using the social capital values. They model the problem of finding influencers in an OSN as a value-allocation problem, where the allocated value represents the individual social capital. In addition, Franks et al. [18] propose a method to identify influential agents in open multi-agent systems by adopting matrix factorization method to measure the influence of nodes in a network.

Summary: The existing methods do not fully consider the social contexts, like social relationships and social trust between participants, and preferences of participants in OSNs. As indicated in Social Psychology [3], [4], [5] and Computer Science [6], such social contexts are significant for social influence evaluation. Therefore, these existing methods cannot deliver accurate social influence results.

\section{PRELIMINARY}

\section{A. Contextual Social Network}

A Contextual Social Network (CSN) [27], [28], [29] is a labeled directed graph $G=(V, E, L V, L E)$, where

- $V$ is a set of vertices; 


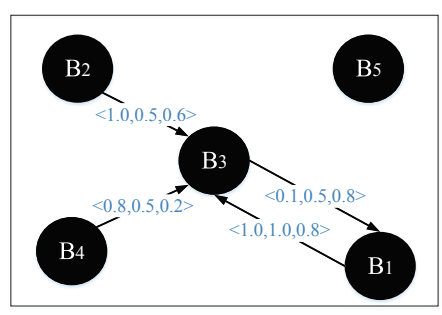

Figure 2. A contextual social network

- $E$ is a set of edges, and $\left(v_{i}, v_{j}\right) \in E$ denotes a directed edge from vertex $v_{i}$ to vertex $v_{j}$;

- $L V$ is a function defined on $V$ such that for each vertex $v$ in $V, L V(v)$ is a set of labels for $v$. Intuitively, the vertex labels may for example represent social roles or social influence in a specific domain;

- $L E$ is a function defined on $E$ such that for each link $\left(v_{i}, v_{j}\right)$ in $E, L E\left(v_{i}, v_{j}\right)$ is a set of labels for $\left(v_{i}, v_{j}\right)$, like social relationships, social trust and preferences in a specific domain.

\section{B. Social Contexts}

Let $P$ denote the set of participants, and $R$ denote the set of social contexts vectors, $\vec{R}<t, s, p>\in R(t, s, p \in$ $[0,1]$ ), where $\vec{R}_{i, j}(t), \vec{R}_{i, j}(s)$ and $\vec{R}_{i, j}(p)$ represent social trust, social relationship and preference similarity between $P_{i}$ and $P_{j}$ respectively. In addition, we use $I N_{i}$ to denote the incoming neighbors of $P_{i}$ and $O N_{i}$ to denote the outgoing neighbors of $P_{i}$.

- Social Trust (ST): Let $t$ denote the trust value between two participants. $\vec{R}_{i, j}(t)=1$ indicates that $P_{i}$ completely trusts $P_{j}$, and $\vec{R}_{i, j}(t)=0$ indicates that $P_{i}$ completely distrusts $P_{j}$.

- Social Relationship (SR): Let $s$ denote the intimacy of the Social Relationship between two participants. $\vec{R}_{i, j}(s)=1$ indicates that $P_{i}$ and $P_{j}$ have intimate social relationship, and $\vec{R}_{i, j}(s)=0$ indicates that $P_{i}$ have not contacted with $P_{j}$.

- Preference Similarity (PS): Let $p$ denote the value of Preference Similarity between two participants. $\vec{R}_{i, j}(p)=1$ indicates that the preferences of $P_{i}$ and $P_{j}$ are exactly the same, and $\vec{R}_{i, j}(p)=0$ indicates that there is nothing in common interest between $P_{i}$ and $P_{j}$.

Although it is difficult to build up comprehensive social trust, social relationship and preference similarity in all domains, it is feasible to build them up in some specific social communities by using data mining techniques [27]. Mining these social contexts' values is another challenging problem, which is out of the scope of this paper.

Example 2: Figure 2 depicts a contextual social network, which contains the social contexts as $\vec{R}_{2,3}=<$

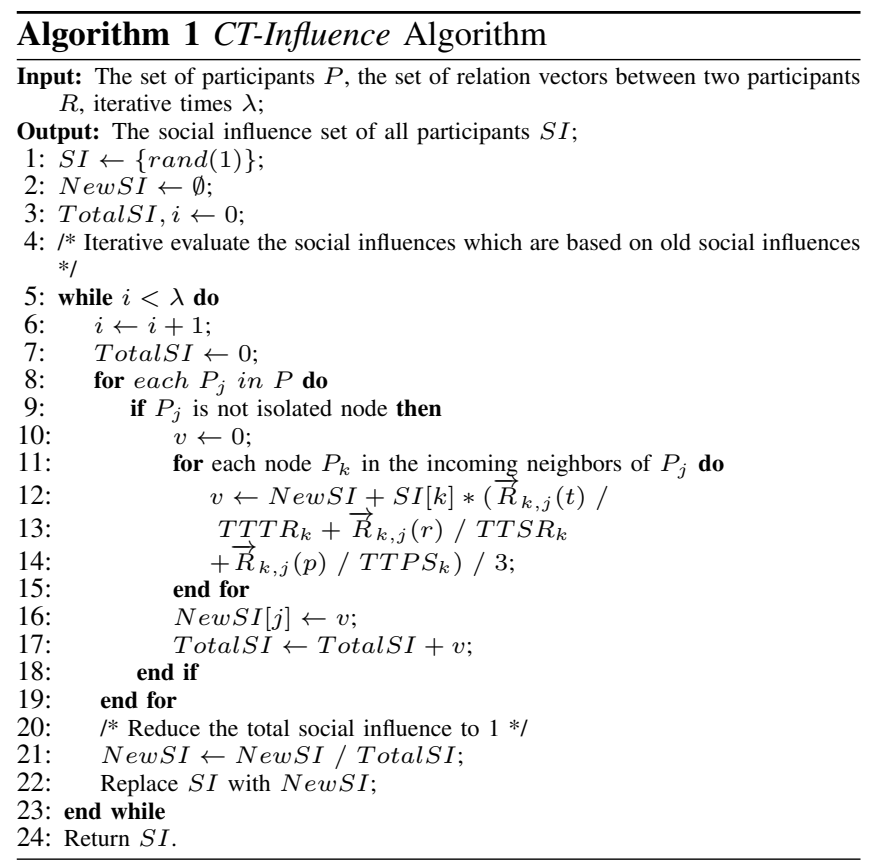

$1.0,0.5,0.6>, \vec{R}_{4,3}=<0.8,0.5,0.2>, \vec{R}_{1,3}=<$ $1.0,1.0,0.8>$ and $\vec{R}_{3,1}=<0.1,0.5,0.8>$.

\section{Context-Aware Trust-Oriented Influencers FINDING METHOD}

In this section, we propose a Context-Aware TrustOriented Influencers Finding method, called CT-Influence, by adopting the iterative method to evaluate social influence. CT-Influence takes the above important social contexts into consideration, and thus can deliver more accurate social influence evaluation results, and therefore can find more reliable Influencers.

\section{A. Algorithm Description}

In our CT-Influence method, the social influence of participants are constantly computed and replaced until the social influences achieve convergence by using iterative method. Next, we introduce the process of iteration and the details of CT-Influence.

The social influences at iteration time $t+1$ are based on the social influences delivered at the last iteration time $t$. In the process of evaluating new social influences, we consider the social contexts ( $S T, S R$ and $P S$ ) between a participant and his/her neighbors equally. Let $S I_{i}^{*}$ denote the social influence of participant $P_{i}$, which can be computed by Eqs. (1) and (2) as below:

$$
S I_{i}^{t+1}=\sum_{P_{k} \in I N_{i}} S I_{k}^{t} \cdot \rho_{k, i}
$$




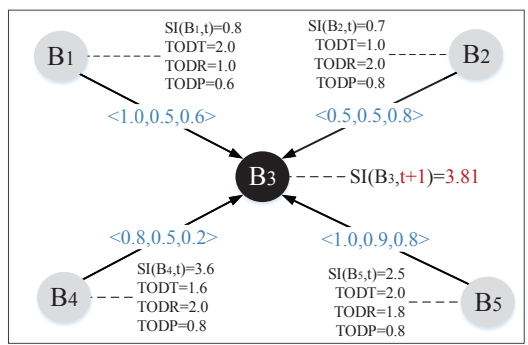

Figure 3. Computing social influence in iterative process

where $\sum_{P_{i} \in O N_{k}} \rho_{k, i}=1$ and

$$
\rho_{k, i}=\frac{\vec{R}_{k, i}(t)}{3 \cdot T T T R_{k}}+\frac{\vec{R}_{k, i}(s)}{3 \cdot T T S R_{k}}+\frac{\vec{R}_{k, i}(p)}{3 \cdot T T P S_{k}} .
$$

$T_{T T R_{k}}=\sum_{P_{j} \in O N_{k}} \vec{R}_{k, j}(t), \quad T T S R_{k}=$ $\sum_{P_{j} \in O N_{k}} \vec{R}_{k, j}(s)$ and

$T T P S_{k}=\sum_{P_{j} \in O N_{k}} \vec{R}_{k, j}(p)$. Here, $\rho_{k, i}$ reflects the whole influence probability from $P_{k}$ to $P_{i}$.

Example 3: The social influence of $P_{1}$ at iteration time $t$ have been shown in Figure 3. Based on Eqs. (1) and (2), at iterative time $t+1$, the social influence $S I_{1}^{t+1}=0.8 \times$ $(1.0 / 2.0+0.5 / 1.0+0.6 / 0.6) / 3+0.7 \times(0.5 / 1.0+0.5 / 2.0+$ $0.8 / 0.8) / 3+3.6 \times(0.8 / 1.6+0.5 / 2.0+0.2 / 0.8) / 3+2.5 \times$ $(1.0 / 2.0+0.9 / 1.8+0.8 / 0.8) / 3=3.81$ (accurate to two decimal places).

\section{B. Convergence of the Iteration}

We use an error function to prove the convergence of our CT-Influence method, which process is similar to the PageRank model [30]. Then we define the total error at iteration time $t$ to be:

$$
\operatorname{Error}(t)=\sum_{i=1}^{N}\left|S I_{i}^{t}-S I_{i}^{*}\right|
$$

where $N$ is the number of participants.

Theorem 1: CT-Influence is convergent, i.e., $\operatorname{Error}(t)<\operatorname{Error}(t-1)$.

Proof 1: Since $S I_{i}^{*}$ is the real solution, according to eq.(1), it must satisfy following equation exactly:

$$
S I_{i}^{*}=\sum_{P_{k} \in I N_{i}} S I_{k}^{*} \cdot \rho_{k, i}
$$

For a participant $P_{i}$, the error at iterative time $t$ is:

$$
S I_{i}^{t}-S I_{i}^{*}=\sum_{P_{k} \in I N_{i}}\left(S I_{k}^{t-1}-S I_{k}^{*}\right) \cdot \rho_{k, i}
$$

Using the Triangle Inequality, we can obtain the expression as follows:

$$
\left|S I_{i}^{t}-S I_{i}^{*}\right| \leq \sum_{P_{k} \in I N_{i}}\left|S I_{k}^{t-1}-S I_{k}^{*}\right| \cdot \rho_{k, i}
$$

Next, we sum all the errors of participants to obtain total error. Notice that $\sum_{P_{i} \in O N_{k}} \rho_{k, i}=1$ :

$$
\begin{aligned}
& \operatorname{Error}(t)=\sum_{i=1}^{N}\left|S I_{i}^{t}-S I_{i}^{*}\right| \\
& \leq \sum_{i=1}^{N} \sum_{P_{k} \in I N_{i}}\left|S I_{k}^{t-1}-S I_{k}^{*}\right| \cdot \rho_{k, i} \\
& =\sum_{\vec{R}_{k, i} \in R}\left|S I_{k}^{t-1}-S I_{k}^{*}\right| \cdot \rho_{k, i} \\
& =\sum_{k=1}^{N}\left|S I_{k}^{t-1}-S I\left(P_{k}\right)^{*}\right| \cdot \sum_{P_{i} \in O N_{k}} \rho_{k, i} \\
& =\operatorname{Error}(t-1)
\end{aligned}
$$

Recalling the eq.(6), we find that $\operatorname{Error}(t)=\operatorname{Error}(t-1)$ if and only if $\forall P_{k} \in P, S I_{k}^{t-1}-S I_{k}^{*}>0$ or $\forall P_{k} \in$ $P, S I_{k}^{t-1}-S I_{k}^{*}<0$. But, our iterative method reduces the total social influence to 1 , which means that $\sum_{k=1}^{N} S I_{k}^{t-1}=$ $\sum_{k=1}^{N} S I_{k}^{*}=1$. It can not satisfy the above condition, so Error $(t)<$ Error $(t-1)$. Then Theorem 1 is proved.

The pseudo-code of the algorithm is given in Algorithm 1. The time complexity of our CT-Influence method is $\mathcal{O}\left(\lambda N^{2}\right)$, where $N$ is the number of participants in an OSN and $\lambda$ is iterative times.

\section{EXPERIMENTS}

In our experiments, we compare our proposed $C T$ Influence method with the state-of-the-art method, SoCap [16] in the accuracy of the two methods in social influence evaluation in Exp-1 and Exp-2. In order to investigate the efficiency of our method, we compare the execution time of the two methods in Exp-3.

\section{A. Experimental Setting}

Table I

EXPERIMENTAL DATASETS

\begin{tabular}{|c|c|c|}
\hline Dataset & Epinions & DBLP \\
\hline Nodes & 75,879 & 317,080 \\
\hline Links & 508,837 & $1,049,866$ \\
\hline Average Indegree & 6.706 & 3.311 \\
\hline High Indegree Nodes (Indegree $\geq 50$ ) & 2032 & 170 \\
\hline The Ratio of High Indegree Nodes & $2.679 \%$ & $0.054 \%$ \\
\hline
\end{tabular}

1) Datasets: We adopt two real social network datasets, Epinions [31] and DBLP [32]. The Epinions dataset has 75,879 nodes and 508,837 links, where each node represents a buyer, and each link corresponds to the relationships between buyers. The DBLP dataset has 317,080 nodes and $1,049,866$ links, where each node represents an author, and each link corresponds to the co-author relationships between authors. The details of the two datasets are listed in Table I. 
2) Ground Truth: As indicated in Social Psychology [33], if a participant can influence the maximal number of participants who have a high social influence, then such a participant has high social influence as well. Therefore, we rank the influencers based on the number of influenced participants as the Ground Truth in Exp-1.

3) Diffusion Models: In Exp-2, we adopt two classical diffusion models, i.e., Linear Threshold (LT) model [34] and Independent Cascade (IC) model [9]. These models have been widely used to investigate the effectiveness of social influence evaluation methods in [35], [36], [37] by comparing the number of nodes that are influenced by the seeds in these diffusion models.

- Linear Threshold (LT) Model: LT model is the first model to imitate the diffusion process of information. The approach is based on the node-specific thresholds [34]. In the model, at time step $t$, all nodes that were influenced in step $t-1$ remain being influenced. A participant $P_{i}$ is influenced based on a monotonic function of its influenced neighbors $f(\operatorname{In}(i, t)) \in[0,1]$ (see Eq.(8)) and a threshold $\theta_{i} \in[0,1]$, i.e., $P_{i}$ is influenced at time $t$ if $f(\operatorname{In}(i, t)) \geq \theta_{i}$.

$$
f(\operatorname{In}(i, t))=\sum_{P_{j} \in \operatorname{In}(i, t)} b_{i, j}
$$

where $\operatorname{In}(i, t)$ is the influenced neighbors of $P_{i}$ at time step $t$. Here, we set

$$
b_{i, j}=\frac{\vec{R}_{i, j}(t)+\vec{R}_{i, j}(s)+\vec{R}_{i, j}(p)}{\sum_{P_{k} \in O n_{i}}\left(\vec{R}_{i, k}(t)+\vec{R}_{i, k}(s)+\vec{R}_{i, k}(p)\right)},
$$

$O n_{i}$ is the outgoing neighbors of $P_{i}$ and $\sum_{P_{j} \in O n_{i}} b_{i, j} \leq 1$. In our experiments, in order to investigate the effectiveness of our method based on different thresholds, for each $P_{i}$, we set $\theta_{i} \in\{0.1,0.3,0.5,0.7,0.9\}$.

- Independent Cascade (IC) Model: IC model is a dynamic cascade model for the diffusion process. The model is based on the interacting particle system from probability theory [9]. At each time step $t$, each participant is either influenced or susceptible. A participant $P_{j}$ that was influenced at time step $t-1$ has a single chance to influence each of its incoming neighbors $P_{i}$. The influence succeeds with probability $p_{i, j}$ (see Eq.(10)). Therefore, for participant $P_{i}$, if at least one of its influenced outgoing neighbors succeeds, $P_{i}$ gets influenced. The probability of participant $P_{i}$ getting influence at time step $t$ is:

$$
f(i, t)=1-\prod_{P_{j} \in \operatorname{In}(i, t-1)}\left(1-p_{i, j}\right)
$$

where $\operatorname{In}(i, t-1)$ is the influenced incoming neighbors of $P_{i}$ at time step $t-1$. Here, we set $p_{i, j}=\left(\vec{R}_{i, j}(t)+\right.$ $\left.\vec{R}_{i, j}(s)+\vec{R}_{i, j}(p)\right) / 3$.

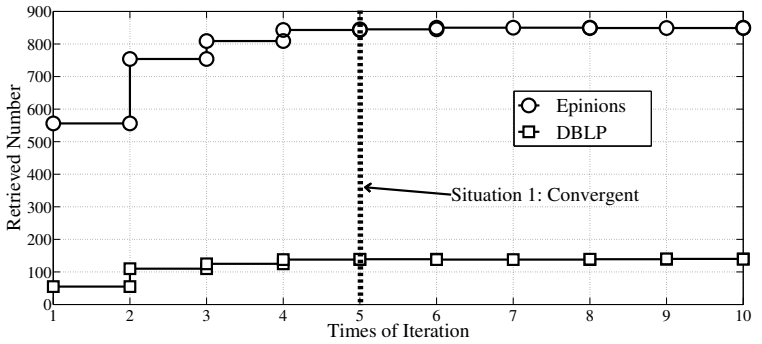

Figure 4. The convergence of our CT-Influence method. $X$ axis is iteration times and $Y$ axis is retrieved number. We use stairs lines to show the trends of retrieved number with the increasing of iteration times.

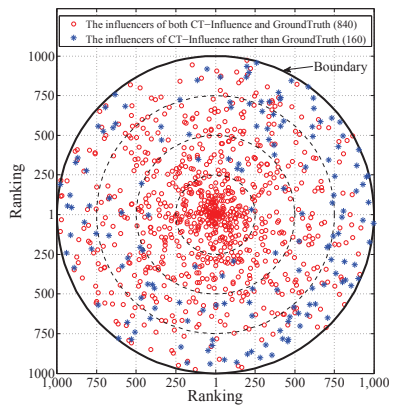

(a) CT-Influence (Epinions)

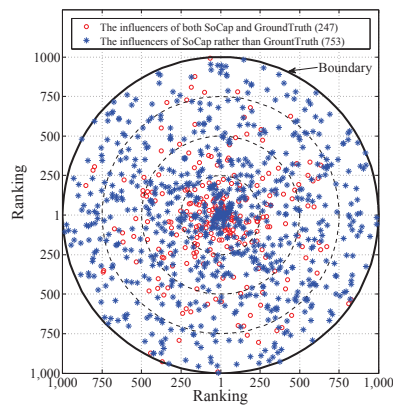

(b) SoCap (Epinions)
Figure 5. Top 1000 influencers delivered by each method on Epinions. The large circle is the boundary which contains the Top-1000 influencers (small red circles and blue stars) delivered by each method. Small red circles are both Ground Truth Top-1000 influencers and Top-1000 influencers delivered by each method respectively. Blue stars are Top-1000 influencers delivered by each method rather than Ground Truth Top-1000 influencers. The more close to the center of the large circle, the higher influence ranking the influencers have.

In our experiments, we select the Top-K influencers delivered by our CT-Influence and SoCap to act as seeds in the different diffusion models respectively, here, $K \in$ $\{1,5,10,20,50,100\}$. Based on the properties of the diffusion models, the number of nodes that are influenced by the seeds delivered by the diffusion models can illustrate the influence of the Top- $K$ influencers [35], [36]. The more the number is, the higher the effectiveness of corresponding method is.

4) Experimental Environments: All experiments were run on a PC powered by two Intel Core i5-3470 CPU 3.20 $\mathrm{GHz}$ processors with $8 \mathrm{~GB}$ of memory, using Windows 7 Professional. The code was implemented by using Visual $\mathrm{C}++2012$ and the experimental data was managed by MySQL Server 5.6. All the experimental results are averaged based on five independent runs.

Table II

THE PERFORMANCES OF CT-Influence AND SoCap with Ground Truth Top-1000

\begin{tabular}{|c|c|c|c|c|}
\hline Method & DataSet & Retrieved Number & Precision & Average Execution Time \\
\hline CT-Influence & Epinions & 840 & 0.84 & $444 \mathrm{~ms}$ \\
\hline SoCap & Epinions & 247 & 0.247 & $6436 \mathrm{~ms}$ \\
\hline CT-Influence & DBLP & 138 & 0.138 & $1244 \mathrm{~ms}$ \\
\hline SoCap & DBLP & 44 & 0.044 & $8364 \mathrm{~ms}$ \\
\hline
\end{tabular}

B. Experimental Results and Analyses 
Table III

THE COMPARISON of CT-Influence AND SoCap with Ground Truth Top-10 ON Epinions

\begin{tabular}{|c|c|c|c|}
\hline Nodes' ID & Ground Truth Ranking & CT-Influence Ranking & SoCap Ranking \\
\hline 18 & 1 & $\mathbf{1}$ & $\mathbf{1 0 8}$ \\
\hline 737 & 2 & $\mathbf{2}$ & $\mathbf{2 6 9}$ \\
\hline 401 & 3 & $\mathbf{3}$ & $\mathbf{3 0 8}$ \\
\hline 40 & 4 & $\mathbf{4}$ & $\mathbf{6 3 1}$ \\
\hline 118 & 5 & $\mathbf{6}$ & $\mathbf{6 6 9}$ \\
\hline 34 & 6 & $\mathbf{7}$ & 1184 (missing) \\
\hline 550 & 7 & $\mathbf{8}$ & 6226 (missing) \\
\hline 136 & 8 & $\mathbf{1 2}$ & 6442 (missing) \\
\hline 143 & 9 & $\mathbf{2 3}$ & 6448 (missing) \\
\hline 1719 & 10 & $\mathbf{3 2}$ & 23842 (missing) \\
\hline
\end{tabular}

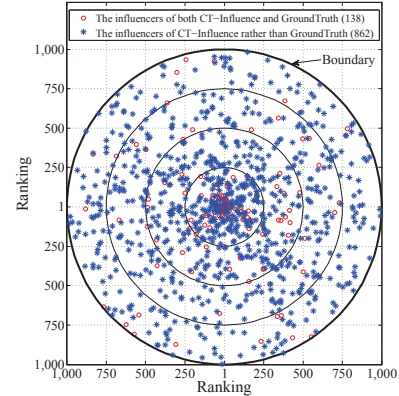

(a) CT-Influence $(D B L P)$

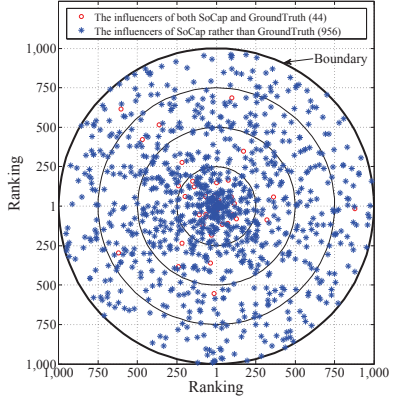

(b) SoCap $(D B L P)$
Figure 6. Top 1000 influencers of delivered by each method on DBLP. X axis and $Y$ axis are all ranking of social influence, and the large circle is the boundary which contains the Top-1000 influencers (small red circles and blue stars) delivered by each method. Small red circles are both Ground Truth Top-1000 influencers and Top-1000 influencers delivered by each method respectively. Blue stars are Top-1000 influencers delivered by each method rather than Ground Truth Top-1000 influencers. The closer to the center of the large circle, the higher influence ranking the influencers have.

1) Exp-1. Effectiveness (by Ground Truth): We measured the precision by varying the Top-1000 influencers retrieved by each method against the Ground Truth Top-1000 influencers.

- Firstly, we observe the trend of Retrieved Number with the increasing of times of iteration to investigate the convergence of our CT-Influence method. Here, Retrieved Number is the number of retrieved influencers, which are both the Ground Truth Top-1000 influencers and the Top-1000 influencers delivered by our CTInfluence method. The experimental results delivered based on Epinions dataset and DBLP datasetare are shown in Figure 4, where we can see that the Retrieved Numbers of our CT-Influence method keep stable after 5 times of iterations for both datasets. Then, in the following experiments, we set the Iterative times $\lambda$ as 5.

- Secondly, after five iterations, the experimental results are listed in Table II. For Epinions, our CT-Influence method finds 840 out of the Ground Truth Top-1000 influencers, while SoCap can only find 247 influencers. Based on the precision function in Eq. (8) [16], the precision of our CT-Influence method is $84 \%$. In contrast, it is only $24.7 \%$ for SoCap method. Therefore, comparing with SoCap, on average, our method greatly improves the precision of social influence evaluation by $240 \%$ in Epinions dataset. For $D B L P$, our $C T$ Influence method finds 138 (precision is $13.8 \%$ ) out of the Ground Truth Top-1000 influencers, but SoCap method only finds out 44 (precision is $4.4 \%$ ). Therefore, on average, our method improves the precision of social influence evaluation by $210 \%$ in $D B L P$ dataset.

$$
\text { Precision }=\frac{\mid \text { Relevant } \bigcap \text { Retrieved } \mid}{\mid \text { Retrieved } \mid}
$$

- Next, we list the results of the Top-10 influencers retrieved by each method against the Ground Truth Top-10 influencers in Table III. From Table III, our CT-Influence method can find all 10 influencers, and the CT-Influence Ranking is very close to the Ground Truth Ranking. But the influencers delivered by SoCap is far away from the Ground Truth Ranking, and 5 out of 10 influencers are missing in the Top-10 list.

- The experimental results of Epinions and DBLP are plotted in Figure 5 and Figure 6, where we can see that the number of the Ground Truth Top-1000 influencers retrieved by our CT-Influence method is more than SoCap's with higher rankings (the small red circles of our CT-Influence method are closer to the center of the large circle). Therefore, our CT-Influence can deliver more accurate social influence evaluation results than SoCap.
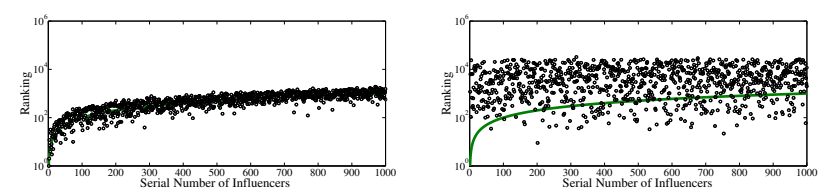

(a)

$A E(C T$

- (b) $A E($ SoCap, Epinions $)=$ Influence, Epinions $)=1713105967223$
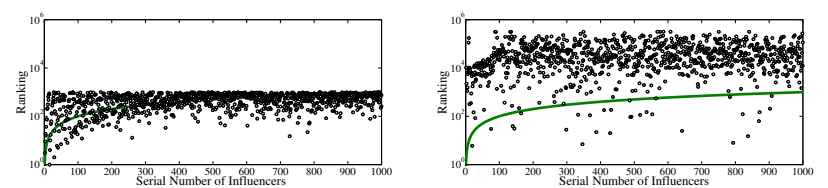

$\begin{array}{lll}\text { (c) } A E(C T & -(\mathrm{d}) & A E(\operatorname{SoCap}, D B L P) \\ \text { Influence, } D B L P)=263866 & 51327091\end{array}$

Figure 7. The accuracy of the two methods on two datasets. $X$ axis is the serial number of influencers, $Y$ axis is the ranking of social influence. Green curve is Ground Truth Ranking, and small black circles are the rankings of influencers delivered by each method.

- In addition, we use an Absolute Error (AE) function to measure the error of each method. The error is the absolute value between the influence ranking delivered by each method and the Ground Truth Ranking. The detailed calculation is as follows:

$$
A E(\text { method }, \text { dataset })=\sum_{P_{i} \in G T}\left|R A\left(P_{i}\right)-G T R A\left(P_{i}\right)\right|
$$

where GT is the set of Ground Truth Top-1000 influencers, $R A\left(P_{i}\right)$ is the influence ranking of $P_{i}$ evaluated 


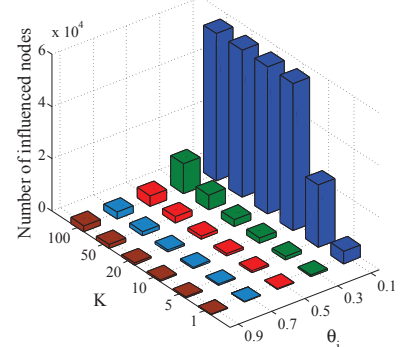

(a) CT-Influence (LT model, Epinions)

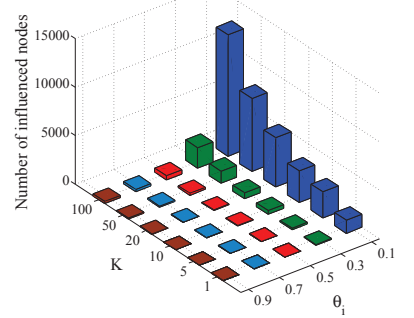

(c) CT-Influence (LT model, DBLP)

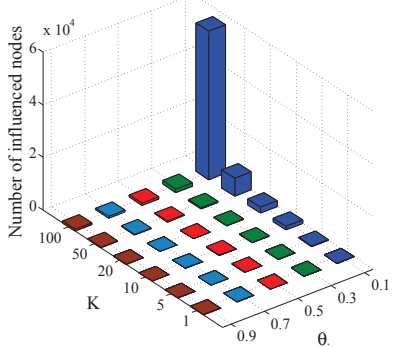

(b) SoCap (LT model, Epinions)

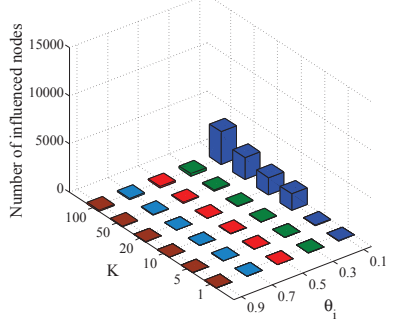

(d) SoCap ( $L T$ model, DBLP)

Figure 8. The number of influenced nodes in Linear Threshold model

by each method, and $G T R A\left(P_{i}\right)$ is the Ground Truth Ranking of $P_{i}$. From Figure 7, AE(SoCap, Epinions) is much greater than $A E(C T-$ Influence, Epinions $)$ and $A E($ SoCap, DBLP) is much greater than $A E(C T$ - Influence, $D B L P)$, so the error level of SoCap is high.

- Finally, we study the accuracy and absolute error of our CT-Influence method by comparing the Top-1000 influencers identified by each method against the Ground Truth Top-1000 influencers. Since SoCap ignore the social relationship and preference similarity between participants, it cannot deliver accurate social influence evaluation results. Therefore, our CT-Influence method outperforms SoCap in Effectiveness based on Ground Truth results.

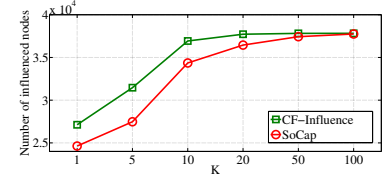

(a) IC model, Epinions

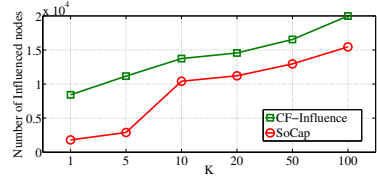

(b) $I C$ model, $D B L P$

Figure 9. The number of influenced nodes in Independent Cascade model

2) Exp-2. Effectiveness (by Diffusion Models): Figure 8 depicts the experimental results of $L T$ model, where we can see that in all cases, the number of influenced nodes identified by our CT-Influence with different $K$ and $\theta_{i}$ are more than that of SoCap. The average number of influenced nodes identified by our CT-Influence is 5,609.18, while that of SoCap is $1,371.02$ which is $75.56 \%$ less than that of CT-Influence. In addition, the number of influenced nodes

identified by the two methods increases with the increase of $K$. This is because that with the increase of $K$, the number of sources for the spread of information increases, which leads to the Top- $K$ influencers identified by both of $C T$ Influence and SoCap can influence more nodes in $L T$ model. Furthermore, the number of influenced nodes identified by the two methods decreases with the decrease of $\theta_{i}$. This is because that the limit for the spread of information decreases with the decrease of $\theta_{i}$, which leads to the Top-K influencers identified by both of CT-Influence and SoCap can influence more nodes in $L T$ model. Therefore, based on the properties of diffusion models [9], the experimental results illustrate that the Top-K influencers identified by our CT-Influence have more influences than that of SoCap in LT model.

Figure 9 depicts the number of influenced nodes identified by our CT-Influence and SoCap, where we can see that with the increase of $K$ in $I C$ model respectively, where we can see that the number of influenced nodes of our CT-Influence are more than that of SoCap in all 6 cases on the two datasets. The average number of influenced nodes identified by our CT-Influence is $24,441.92$, and that of SoCap is $21,069.5$ which is $13.8 \%$ less than the former. This is because that based on the properties of the IC model introduced in the Section Diffusion Models, with taking the three social contexts into consideration, the Top- $K$ influencers identified by our CT-Influence have higher probability to influence their neighbor nodes. In addition, with the increase of $K$, the number of nodes influenced by the Top-K nodes identified by both of the two methods increases.

From the experimental results in the two classical diffusion models, i.e., $L T$ model and $I C$ model, we can see that the Top-K influencers identified by our CT-Influence have more influences than that of the state-of-the-art method, SoCap. Based on the properties of diffusion models, on average, our CT-Influence improves the effectiveness of SoCap by $90 \%$. Thus our CT-Influence method outperforms SoCap in effectiveness based on the two classical diffusion models.

3) Exp-3. Efficiency: Table II lists the corresponding execution times of social influence evaluation (except the time of "loading all data into memory") of two methods. On Epinions dataset, the average execution time is $444 \mathrm{~ms}$ for our CT-Influence. By contrast, it is $6,436 \mathrm{~ms}$ for SoCap. On average, our method can save $93.1 \%$ of the execution time. On DBLP dataset, it is $1,244 \mathrm{~ms}$ for our CT-Influence and 8,364 ms for SoCap. On average, our method can save $85.1 \%$ of the execution time. This is because that based on Theorem 1, the convergence of our CT-Influence is fast. Therefore, our CT-Influence method greatly outperforms SoCap in efficiency.

\section{CONCLUSION}

In this paper, we have proposed a Context-Aware TrustOriented Influencers Finding (CT-Influence) method based 
on the social trust, the social relationships and the preference similarity between two participants to evaluate the social influences. The experiments conducted on two real social network datasets (Epinions and DBLP) have demonstrated our CT-Influence method greatly outperforms the state-ofthe-art method, SoCap, and can deliver more accurate social influence evaluation results with less execution time.

\section{ACKNOWLEDGEMENT}

This work was partially supported by Natural Science Foundation of China (Grant Nos. 61303019, 61402312, 61402313, 61232006, 61003044, 61440053), Doctoral Fund of Ministry of Education of China (20133201120012), and Collaborative Innovation Center of Novel Software Technology and Industrialization, Jiangsu, China.

\section{REFERENCES}

[1] A. Liu, Q. Li, X. Zhou, L. Li, G. Liu, and Y. Gao, "Rating propagation in web services reputation systems: A fast shapley value approach," in DASFAA' 14,2014 , pp. 466-480.

[2] A. Liu, Q. Li, L. Huang, S. Ying, and M. Xiao, "Coalitional game for community-based autonomous web services cooperation," IEEE T. Services Computing, vol. 6, no. 3, pp. 387$399,2013$.

[3] E. Berscheid, H. T. Reis et al., "Attraction and close relationships," The handbook of social psychology, vol. 2, pp. 193-281, 1998.

[4] I. Yaniv, "Receiving other peoples advice: Influence and benefit," Organizational Behavior and Human Decision Processes, vol. 93, no. 1, pp. 1-13, 2004.

[5] S. T. Fiske, Social beings: Core motives in social psychology. John Wiley \& Sons, 2009.

[6] P. Bedi, H. Kaur, and S. Marwaha, "Trust based recommender system for semantic web." in IJCAI, 2007, pp. 2677-2682.

[7] Z. Li, L. Sitbon, L. Wang, X. Zhou, and X. Du, "Aml: Efficient approximate membership localization within a webbased join framework," Knowledge and Data Engineering, IEEE Transactions on, vol. 25(2), pp. 298-310, 2013.

[8] Z. Li, M. A. Sharaf, L. Sitbon, X. Du, and X. Zhou, "Core: a context-aware relation extraction method for relation completion," Knowledge and Data Engineering, IEEE Transactions on, vol. 26, no. 4, pp. 836-849, 2014.

[9] D. Kempe, J. Kleinberg, and É. Tardos, "Maximizing the spread of influence through a social network," in $K D D, 2003$, pp. 137-146.

[10] W. Chen, Y. Wang, and S. Yang, "Efficient influence maximization in social networks," in $K D D, 2009$, pp. 199-208.

[11] K. Jung, W. Heo, and W. Chen, "Irie: Scalable and robust influence maximization in social networks," in ICDM, 2012, pp. $918-923$.

[12] W. Chen, W. Lu, and N. Zhang, "Time-critical influence maximization in social networks with time-delayed diffusion process." in AAAI, 2012, pp. 592-598.

[13] W. Chen, C. Wang, and Y. Wang, "Scalable influence maximization for prevalent viral marketing in large-scale social networks," in $K D D, 2010$, pp. 1029-1038.

[14] J. Kim, S.-K. Kim, and H. Yu, "Scalable and parallelizable processing of influence maximization for large-scale social networks?" in ICDE, 2013, pp. 266-277.
[15] J. Leskovec, A. Krause, C. Guestrin, C. Faloutsos, J. VanBriesen, and N. Glance, "Cost-effective outbreak detection in networks," in $K D D, 2007$, pp. 420-429.

[16] K. Subbian, D. Sharma, Z. Wen, and J. Srivastava, "Finding influencers in networks using social capital," in ASONAM, 2013, pp. 592-599.

[17] C.-m. Au Yeung and T. Iwata, "Strength of social influence in trust networks in product review sites," in WSDM, 2011, pp. 495-504.

[18] H. Franks, N. Griffiths, and S. S. Anand, "Learning influence in complex social networks," in $A A M A S, 2013$, pp. 447-454.

[19] J. Guo, P. Zhang, C. Zhou, Y. Cao, and L. Guo, "Personalized influence maximization on social networks," in CIKM, 2013, pp. 199-208.

[20] K. Kutzkov, A. Bifet, F. Bonchi, and A. Gionis, "Strip: stream learning of influence probabilities," in $K D D, 2013$, pp. 275 283.

[21] K. Subbian, C. C. Aggarwal, and J. Srivastava, "Contentcentric flow mining for influence analysis in social streams," in $C I K M, 2013$, pp. 841-846.

[22] R. Mansell and B. S. Collins, Introduction: Trust and crime in information societies. Edward Elgar, 2005.

[23] S. S. Brehm, Intimate relationships. Mcgraw-Hill Book Company, 1992.

[24] N. Luhmann, H. Davis, J. Raffan, and K. Rooney, Trust; and, Power: two works by Niklas Luhmann. Wiley Chichester, 1979.

[25] F. Chua and E.-P. Lim, "Trust network inference for online rating data using generative models," in $K D D^{\prime} 10$, pp. 889 898.

[26] T. Iwata, A. Shah, and Z. Ghahramani, "Discovering latent influence in online social activities via shared cascade poisson processes," in KDD, 2013, pp. 266-274.

[27] G. Liu, Y. Wang, and M. A. Orgun, "Trust transitivity in complex social networks." in $A A A I$, vol. 11, 2011, pp. 1222 1229.

[28] G. Liu, K. Zheng, Y. Wang, M. A. Orgun, A. Liu, L. Zhao, and X. Zhou, "Multi-constrained graph pattern matching in large-scale contextual social graphs," in ICDE'15, 2015, pp. $351-362$

[29] G. Liu, Y. Wang, and M. A. Orgun, "Optimal social trust path selection in complex social networks," in $A A A I ' 10,2010$, pp. 1391-1398.

[30] T. Haveliwala, "Efficient computation of pagerank," 1999.

[31] M. Richardson, R. Agrawal, and P. Domingos, "Trust management for the semantic web," in ISWC, 2003.

[32] J. L. Jaewon Yang, "Defining and evaluating network communities based on ground-truth," in ICDM, 2012.

[33] R. J. Deluga, "The relation between trust in the supervisor and subordinate organizational citizenship behavior." Military Psychology, vol. 7, no. 1, p. 1, 1995.

[34] M. Granovetter, "Threshold models of collective behavior," American journal of Sociology, vol. 83, no. 6, pp. 1420-1443, 1978.

[35] S. Feng, X. Chen, G. Cong, Y. Zeng, Y. M. Chee, and Y. Xiang, "Influence maximization with novelty decay in social networks," in AAAI, 2014, pp. 37-43.

[36] M. Purohit, B. A. Prakash, C. Kang, Y. Zhang, and V. S. Subrahmanian, "Fast influence-based coarsening for large networks," in $K D D, 2014$, pp. 1296-1305.

[37] A. Srivastava, C. Chelmis, and V. K. Prasanna, "Influence in social networks: A unified model?" in ASONAM, 2014, pp. $451-454$. 